\title{
A Single Therapeutic Echo Endoscope Used for Combined Transgastric and Transpapillary Drainage of a Pancreatic Abscess
}

The design of endoscopic ultrasound (EUS) instruments has been improved to provide a wide therapeutic channel and an elevator to facilitate the movement of needles and medical devices. These improvements make it possible to carry out combined ultrasound and endoscopic approaches in the pancreas and duodenal papilla, with ultrasound examination of surrounding fluid collections [1-5]. Cystogastrostomy is a particularly interesting field for therapeutic endosonography, with major advantages over conventional therapeutic endoscopy - including increased safety, access to distant collections, and differentiation of cystic lesions [5]. Although cystoenterostomy is an effective form of treatment, it does not provide treatment for impaired ductal drainage [3]. We report here on the use of a combined EUS and endoscopic retrograde cholangiopancreatography procedure using a single echo endoscope.

A 39-year-old patient receiving hemodialysis presented with pain and fever and a large infected collection around the pancreatic tail, several months after an episode of biliary acute necrotizing pancreatitis. Cystogastrostomy was initially carried out under EUS guidance using a therapeutic Pentax EG-3830-UT echo endoscope (Pentax Europe, Hamburg, Germany) connected to an EUB-6500 processor (Hitachi Medical Systems, Heverlee, Belgium). A 19-gauge needle (Wilson-Cook EUSN-19-T, Cook Ireland Ltd., Limerick, Ireland) was used to puncture the abscess and fill the collection with contrast medium after microbacterial sampling (Figure [1]). A 0.035-in Jagwire (Boston Scientific, Maastricht, The Netherlands) was inserted into the collection, and the cystogastrostomy was enlarged with a 6-Fr and 8.5-Fr diathermic sheath (Boucard, Uccle, Belgium) in order to place a $4-\mathrm{cm}$ 10-Fr double-pigtail stent (RMS, Lennik, Belgium). The procedure was immediately followed by transpapillary pancreatography and pancreatic sphincterotomy with the same echo endoscope (Figure [2]). Access to the papilla and the pan-
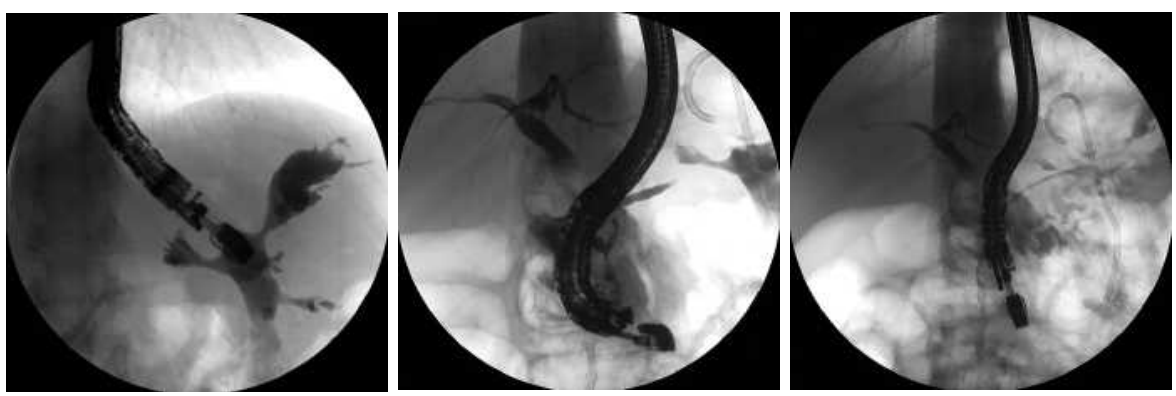

Figure 1 Transgastric drain- Figure 2 Biliopancreatogra- Figure 3 Combined transgasage of a pancreatic abscess phy carried out with the side- tric (4-cm 10-Fr pigtail) and with a linear-array therapeutic viewing echo endoscope be- transpapillary (7-Fr Zimmon) echo endoscope. fore pancreatic sphincterot- stenting, carried out with a sinomy and placement of a pan- gle therapeutic echo endocreatic stent. scope.

creatic duct was easily achieved using this $50^{\circ}$ oblique therapeutic endoscope with a $120^{\circ}$ viewing angle. However, some expertise is needed due to its longer and stiffer end in comparison with a duodenoscope. A large fistula was observed in the caudal pancreatic duct and was treated by placement of a pancreatic Zimmon 7-Fr 8cm stent (Cook, Strombeek-Bever, Belgium) (Figure [3]). The patient was discharged the day after the procedure without complications. Antibiotics were administered for 5 days, and the pancreatic stent was extracted 3 months later, with complete resolution of the fistula and fluid collection.

\section{Benyoumes, P. H. Deprez}

Digestive Endoscopy Unit, Dept. of

Gastroenterology, St.-Luc University Hospital, Catholic University of Louvain, Louvain, Belgium.

\section{References}

${ }^{1}$ Bataille L, Deprez P. A new application for therapeutic EUS: main pancreatic duct drainage with a "pancreatic rendezvous technique". Gastrointest Endosc 2002; 55: 740-743

${ }^{2}$ Sanchez Cortes E, Maalak A, Le Moine Oet al. Endoscopic cystenterostomy of nonbulging pancreatic fluid collections. Gastrointest Endosc 2002; 56: $380-$ 386
${ }^{3}$ Baron TH, Harewood GC, Morgan DE, Yates MR. Outcome differences after endoscopic drainage of pancreatic necrosis, acute pancreatic pseudocysts, and chronic pancreatic pseudocysts. Gastrointest Endosc 2002; 56: 7 - 17

${ }^{4}$ Giovannini M, Bories E, Moutardier Vet al. Drainage of deep pelvic abscesses using therapeutic echo endoscopy. Endoscopy 2003; 35: $511-514$

${ }^{5}$ Giovannini M, Pesenti C, Rolland ALet al. Endoscopic ultrasound-guided drainage of pancreatic pseudocysts or pancreatic abscesses using a therapeutic echo endoscope. Endoscopy 2001; 33: $473-477$

\section{Corresponding Author}

\section{P. Deprez, M.D.}

Digestive Endoscopy Unit, Dept. of Gastroenterology Cliniques Universitaires St.-Luc Catholic University of Louvain Avenue Hippocrate 10 1200 Brussels

Belgium

Fax: $\quad+32-2-7648927$

E-mail: deprez@gaen.ucl.ac.be 